\title{
Eczema Crónico das Mãos Relacionado com Alergia de Contacto a Lactonas Sesquiterpénicas e Partenolido
}

\author{
Leonor Lopes', A I. Gouveia', Teresa E. Correia² \\ 'Interna do Internato Complementar de Dermatologia e Venereologia/Resident, Dermatology and Venereology, Clinica Universitária \\ de Dermatologia, Hospital de Santa Maria (Centro Hospitalar Lisboa Norte - CHLN), Lisboa, Portugal \\ 'Assistente Hospitalar Graduada de Dermatologia e Venereologia/Senior Consultant, Dermatology and Venereology, Clinica \\ Universitária de Dermatologia, Hospital de Santa Maria (CHLN), Lisboa, Portugal
}

RESUMO - As dermatites de contacto alérgicas por plantas podem manifestar-se sob padrões clínicos diversos.

Descrevemos o caso clínico de doente do sexo feminino, de 63 anos com eczema das mãos. Perante a suspeita clínica de dermatite de contacto alérgica efectuaram-se provas epicutâneas com as séries básica (Grupo Português de Estudo das Dermites de Contacto - GPEDC) e de plantas, que revelaram positividades para a mistura de lactonas sesquiterpénicas $0,1 \%$ vas (+ + ) e partenolido $0,1 \%$ vas. $(++)$ em D2 e D4, o que permitiu o diagnóstico de alergia do contacto ao partenolido relacionada com a exposição ocupacional à planta margaça-de-inverno (Chamaemelum fuscatum) nas atividades de jardinagem.

Salienta-se a importância de uma anamnese exaustiva para aumentar o nível de suspeição de dermatite de contacto alérgica e avaliar a relevância dos testes positivos a lactonas sesquiterpénicas e ao partenolido, como agentes etiológicos em casos de eczema crónico das mãos.

PALAVRAS-CHAVE - Dermatite Alérgica de Contacto; Dermatoses da Mão; Extractos Vegetais; Plantas; Sesquiterpenos; Tanacetum parthenium.

\section{Chronic Hand Eczema Due to Contact Allergy to Sesquiterpene Lactones and Parthenolide}

ABSTRACT - Allergic contact dermatitis to plants can assume different clinical patterns.

We report a 63-year-old female patient with chronic hand dermatitis. Given the clinical suspicion of allergic contact dermatitis, patch tests were performed with the Baseline series of the GPEDC and a plants series which showed positivity to sesquiterpene lactone mix $0.1 \%$ pet $(++)$ and parthenolide $0.1 \%$ pet $(++)$ at day D2 and D4. These test results allowed the diagnosis of contact allergy to parthenolide that was related to occupational exposure Chamaemelum fuscatum during gardening.

We emphasize the importance of a meticulous clinical history to increase the level of suspicion of Allergic contact dermatitis and evaluate the relevance of a positive patch test to sesquiterpene lactone and parthenolide as the etiology in chronic hand dermatitis. KEY-WORDS - Dermatitis, Allergic Contac; Hand Dermatoses; Plant Extracts; Plants; Sesquiterpenes; Tanacetum parthenium.

\section{INTRODUÇÃO}

As plantas podem causar uma variedade de reações cutâneas adversas como dermites de contacto alérgica (DCA) e irritativa, urticária de contacto e fitofotodermatites, em diferentes ambientes ocupacionais e profissionais. ${ }^{1}$

As DCA por plantas podem assumir padrões clínicos diversos, sendo os mais frequentes eczema das mãos e pulpites, dermatite fotoalérgica, dermatite aerotransportada ou eczema agudo e subagudo na área de contacto directo com a planta. ${ }^{2}$ Como a DCA ao partenolido tem manifestações clínicas polimorfas é importante considerar esta patologia, por vezes sub-diagnosticada, em doentes com exposição ocupacional a este alergeno. ${ }^{3}$
Correspondência: Leonor Lopes

Serviço de Dermatologia e Venereologia - Centro Hospitalar Lisboa Norte - Hospital de Santa Maria

Avenida Professor Egas Moniz - 1649-035 Lisboa, PORTUGAL

Tel.: 00351217805197 - Fax: 00351217954447

E-mail: leonorlopes@gmail.com
Recebido/Received

22 Abril/22 April 2016

Aceite/Accepted

4 Julho/4 July 2016 


\section{GPDEC}

\section{CASO CLÍNICO}

Doente do sexo feminino, caucasiana, 63 anos, reformada, referenciada à consulta de dermatologia de contacto por dermatose monomorfa simétrica com 3 anos de evolução, caraterizada por placas eritemato-descamativas, hiperqueratósicas e fissuradas, pruriginosas, afectando, preferencialmente, as palmas e polpas dos dedos, com maior intensidade na mão dominante na área palmar e no primeiro dedo de ambas as mãos (Fig.1). Negava antecedentes pessoais ou familiares de atopia e psoríase. A actividade ocupacional principal era doméstica, mas dedicava-se, regularmente, à agricultura e jardinagem.

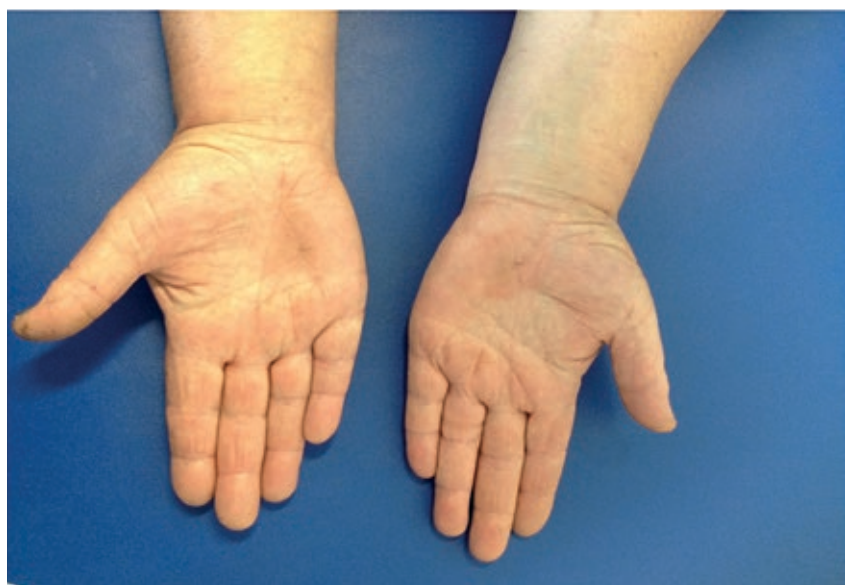

Figura 1 - Eczema crónico das mãos caraterizado por placas hiperqueratósicas e fissuradas, pruriginosas, localizadas nas palmas e polpas dos dedos, com maior intensidade na mão dominante (área palmar) e no primeiro dedo de ambas as mãos.

Perante a suspeita clínica de DCA efectuaram-se provas epicutâneas com a série básica do GPEDC que revelaram positividade para a mistura de lactonas sesquiterpénicas $0,1 \%$ vas. (++) D2 e D4 (Fig. 2a). Face a este resultado testou-se a série de plantas (chemotechnique ${ }^{\circledR}$ ) com positividade para $\circ$ partenolido $0,1 \%$ vas. $(++)$ em D2 e D4 (Fig. 2 b).

Neste caso clínico a sensibilização às lactonas sesquiterpénicas e ao partenolide deveu-se ao contacto directo com a planta margaça-de-inverno (Chamaemelum fuscatum ${ }^{4}$ ), identificada pela doente, na sua actividade ocupacional (Fig. 3). Esta planta da família das asteráceas é originária da região mediterrânica ocidental e pode encontrar-se em todo o país, em campos cultivados ou de pousio que mantenham bastante humidade. Dado o seu longo período de floração (entre novembro e julho) esta planta é muito infestante.

A doente foi aconselhada a lavar frequentemente as mãos com água e sabão depois da sua actividade na agricultura e jardinagem, utilizar roupas protetoras e luvas de nitrilo (uma vez que as lactonas sesquiterpénicas são permeáveis ao vinil, polietileno ou látex). Foi também aconselhada a evitar o contacto com produtos de naturopatia e cosmética nos quais estes alérgenos possam existir. Na consulta de seguimento a

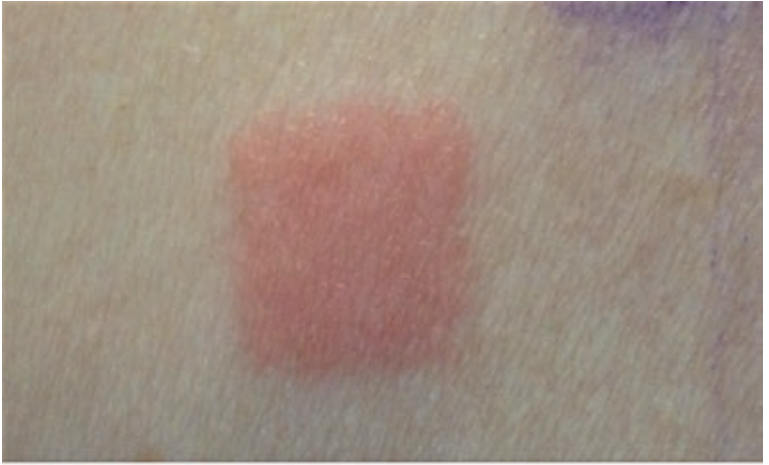

Mist. LS 0,1\%

D4

Figura 2a - Positividade à mistura de lactonas sesquiterpénicas 0, $1 \%$ vas. $(++)$ em D4.

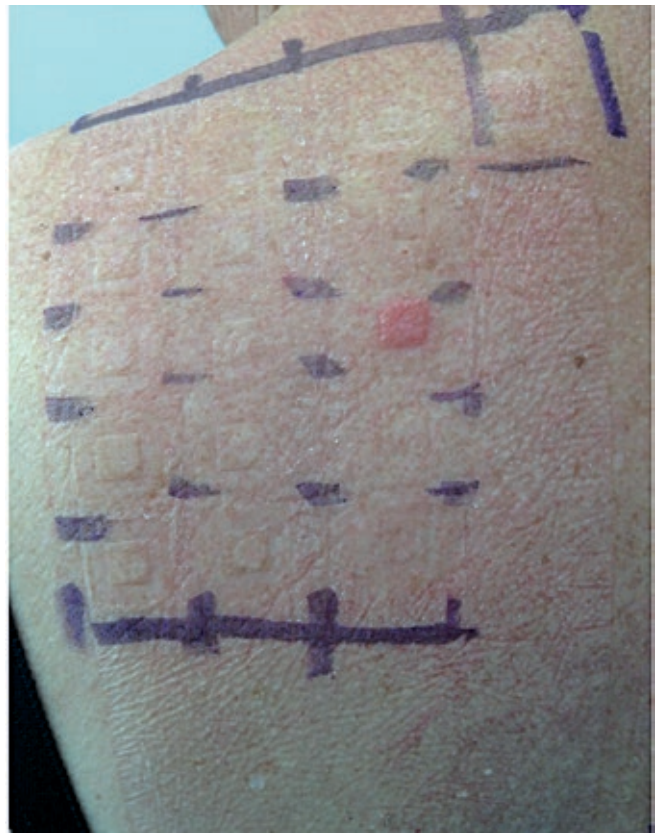

Figura $\mathbf{2 b}$ - Testes epicutâneos com a série de plantas - Positividade ao partenolido $0,1 \%$ vas. $(++)$ em D2.

doente referiu uma melhoria marcada da dermatose com a evicção da planta Margaça-de-Inverno e de outras plantas da família das compostas.

\section{DISCUSSÃO}

As plantas da família das Compositae ou Asteraceae são a segunda maior família de plantas com flor do mundo, ${ }^{5}$ contendo mais de 20000 espécies. Estas podem ser classificadas em: ornamentais, comestíveis, medicinais, aromáticas e condimentares. São alguns exemplos de plantas desta família: o crisântemo, a arnica, o girassol, a matricária, a camomila, a alface e a chicória. A dermite pelas compostas é uma das causas mais frequentes de DCA por plantas, afetando 


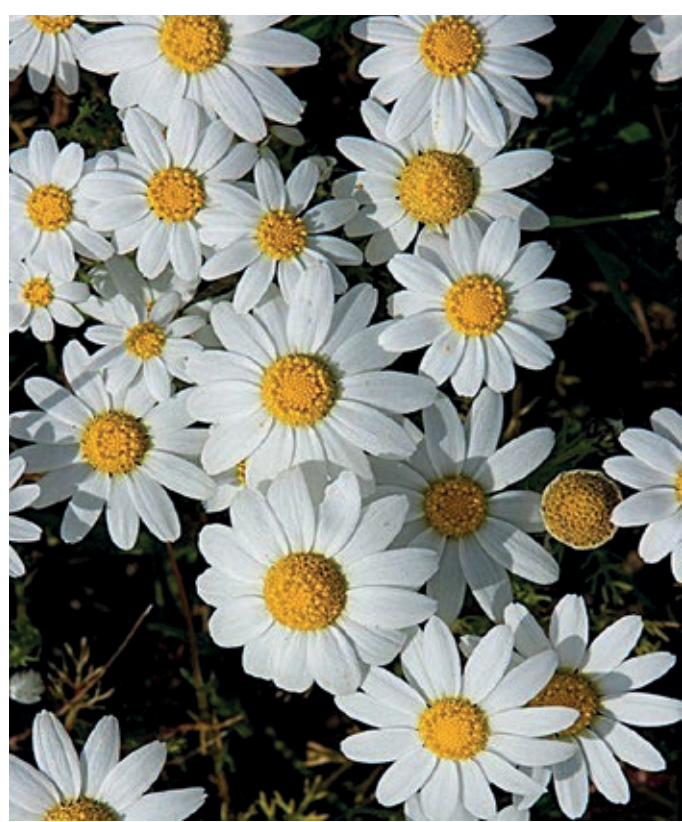

Figura 3 - Planta margaça-de-inverno (Chamaemelum fuscatum ${ }^{4}$ ).

0,7-1,4\% da população em geral e $4,5 \%$ dos grupos expostos de forma ocupacional. ${ }^{5}$

Os principais alérgenos causadores de eczemas por hipersensibilidade de contacto retardada nas plantas são as lactonas sesquiterpénicas, presentes em especial na família das Compositae mas ainda noutras famílias como: Lauraceae, Magnolíaceae e Jubulaceae. ${ }^{2}$ As lactonas sesquiterpénicas têm em comum, na porção antigénica, um anel de alfa-metileno- $Y$-butirolactona, ${ }^{2,6}$ mas existem diferentes lactonas sesquiterpénicas que podem ter reacções cruzadas entre si ou pode ocorrer sensibilização concomitante devido à exposição simultânea a diferentes lactonas no contacto com estas plantas. $O$ partenolido é um forte sensibilizante implicado na alergia a plantas ${ }^{3}$ e é a principal lactona sesquiterpénica (compreende $85 \%$ do total) existente no Tanacetum parthenium ou matricária. ${ }^{5} \mathrm{~A}$ planta identificada pela doente, margaça-de-inverno, ${ }^{4}$ é da mesma família das compostas o que explica a positividade à mistura de lactonas sesquiterpénicas. A positividade ao partenolido pode ser atribuída a uma reação cruzada entre diferentes lactonas sesquiterpénicas ou a co-sensibilização.

O número de DCA ao partenolido pode aumentar pela sua utilização em naturopatia e cosmética no "tratamento" da artrite, asma, obstipação, cefaleias, cólicas, vertigens, entre outros. ${ }^{5}$ A aplicação diária de cremes contendo partenolido pode ser suficiente para causar DCA e pode ser agravado pela exposição externa ("airborne") a este alérgeno., 6 "No entanto, a concentração do partenolido nestes produtos varia consoante as partes da planta utilizadas na sua composição, e a sua estabilidade varia com a fórmula farmacêutica utilizada (solução ou pó) e com alterações da temperatura e da humidade. ${ }^{7}$ As formulações tópicas contendo partenolido podem provocar provas epicutâneas de contacto positivas em doentes alérgicos à matricária. Porém, a sua reatividade é perdida ao longo do tempo, o que pode explicar algumas provas epicutâneas negativas. ${ }^{7}$ A nossa doente não utilizava nenhum produto de aplicação tópica contendo este alérgeno.

O estudo da DCA por plantas nem sempre é fácil porque muitos doentes não consideram essa possibilidade, e torna-se difícil identificar o agente causal devido ao contacto simultâneo com múltiplas plantas. ${ }^{2} \mathrm{~A}$ anamnese exaustiva é imprescindível para aumentar o nível de suspeição desta etiologia, nomeadamente em casos de eczema crónico das mãos. Na história clínica é muito importante interrogar e avaliar a presença de lesões agudas com padrão aerotransportado, lineares ou hiperqueratósicas e fissuradas das polpas e palmas das mãos. ${ }^{2} \mathrm{~A}$ doente negou sempre a presença de lesões com padrão aerotransportado ou noutras localizações com excepção das mãos.

Na série básica do GPEDC é a mistura de lactonas sesquiterpénicas que nos orienta para o diagnóstico de DCA por plantas. Esta é constituída por alantolactona 0,033\% vas., costunolido $0,033 \%$ vas. e dehidrocostus lactona $0,033 \%$ vas. (chemotechnique ${ }^{\circledR}$ ). A mistura de lactonas sesquiterpénicas é responsável por $1,4-1,8 \%$ das provas de contacto positivas ${ }^{8}$ e a prevalência de sensibilização ocupacional ronda os 11 19\%. ${ }^{9}$ A sua inclusão na série básica é justificada por uma taxa de cerca de $65 \%$ de testes relevantes, rara sensibilização activa e reações irritativas desprezíveis. ${ }^{9}$ No entanto, como não é uma mistura muito sensível devem testar-se alérgenos complementares. ${ }^{2,8} \mathrm{~A}$ mistura de compostas é um marcador de rastreio mais sensível ${ }^{10}$ obtendo prevalências entre os 2,3$3,1 \%$ e uma sensibilização ocupacional de $28 \% .^{9-10}$ Sendo obtida a partir de extractos de plantas, esta mistura pode ter alguma variabilidade relacionada com o conteúdo de cada planta, e ainda, do conjunto de plantas selecionadas pelo fornecedor para incluir na mistura, que contém habitualmente extratos de Arnica montana, Chamomilla recutita, Achillea millefolium, Tanacetum vulgare, Anthemis nobilis e partenolido, em duas concentrações distintas ( $5 \%$ e 2,5\% vas). Quanto maior a concentração desta mistura, maior a sua taxa de detecção. Porém, a concentração mais baixa permite reduzir a irritação local e o risco de sensibilização activa, mantendo uma sensibilidade superior à mistura de lactonas sesquiterpénicas. Como existem doentes com provas epicutâneas positivas para apenas uma das misturas (mistura de lactonas sesquiterpénicas ou mistura de compostas), Bong e colaboradores recomendam que sejam realizadas provas epicutâneas com as duas misturas de forma a atingir uma taxa de deteção global superior a 90\%. ${ }^{9}$ Todavia, Paulsen defende que a mistura de lactonas sesquiterpénicas tem uma sensibilidade suficiente para ser utilizada como marcador de rastreio isolado, devendo apenas ser suplementada com a mistura de compostas em caso de necessidade. ${ }^{9}$

Quando não se dispõe dos alérgenos específicos para a suspeita clínica e não se trata de uma planta irritativa podem fazer-se provas epicutâneas com a própria planta (flores, caules, raízes e folhas fragmentadas a fresco, congeladas ou secas) misturada com água e vaselina ou com extratos da planta em acetona, éter ou álcool. ${ }^{2,5}$ Estas provas são muito úteis, 
mas podem induzir reacções irritativas, falsamente positivas, ou podem sensibilizar os doentes quando se utilizam concentrações altas dos seus extratos (> 1\%). ${ }^{2,5}$ No nosso caso clínico não foi possível realizar provas de contacto com a planta específica uma vez que não foi possível obter a planta fresca.

Conflitos de interesse: Os autores declaram não possuir conflitos de interesse. Suporte financeiro: $O$ presente trabalho não foi suportado por nenhum subsídio ou bolsa.

Confidencialidade dos dados: Os autores declaram ter seguido os protocolos do seu centro de trabalho acerca da publicação dos dados de doentes.

Conflicts of interest: The authors have no conflicts of interest to declare. Financing Support: This work has not received any contribution, grant or scholarship.

Confidentiality of data: The authors declare that they have followed the protocols of their work center on the publication of data from patients.

\section{REFERÊNCIAS}

1. Jean L. Bolognia, Dermatology. 3rd ed. Amsterdam: Elsevier; 2012.

2. Gonçalo M. Dermatosis profesionales por plantas e maderas. In: Conde-Salazar Gómez L, Ancona Alayón A, editores. Dermatosis Profesionales. Barcelona: Menarini Área Científica; 2000. p.121-8.
3. De D, Jindal R, Kanwar AJ. Contact dermatitis to parthenium simulating lichen nitidus. Indian J Dermatol Venereol Leprol 2010; 76:286-8.

4. Chamaemelum fuscatum [consultado a 21 de Abril de 2016]. Disponível em: http://flora-on.pt/index. php? $q=$ Chamaemelum + fuscatum

5. Sharma VK, Verma P. Parthenium dermatitis in India: Past, present and future. Indian J Dermatol Venereol Leprol 2012; 78:560-8.

6. Killoran C. Two cases of compositae dermatitis exacerbated by moisturizer containing feverfew. Dermatitis. 2007; 18:225-9.

7. Paulsen E, Christense LP, Fretté XC, Andersen KE. Patch test reactivity to feverfew - containing creams in feverfew allergic patients. Contact Dermatitis 2010; 63:146-50.

8. Orion, E, Paulsen E, Andersen KE, Menné T. Comparison of simultaneous patch testing with parthenolide and sesquiterpene lactone mix. Contact Dermatitis. 1998; 38:207-8.

9. Paulsen E, Andersen KE, Hausen BM. An 8-year experience with routine SL mix patch testing supplemented with Compositae mix in Denmark. Contact Dermatitis. 2001; 45:29-35.

10. Bong JL, English JS, Wilkinson SM. Diluted Compositae mix versus sesquiterpene lactone mix as a screening agent for Compositae dermatitis: a multicentre study. Contact Dermatitis. 2001 ; 45:26-8. 